\title{
ON SATURATION AND THE MODEL THEORY OF COMPACT KÄHLER MANIFOLDS
}

\author{
RAHIM MOOSA
}

\begin{abstract}
A hypothesis is introduced under which a compact complex analytic space, $X$, viewed as a structure in the language of analytic sets, is essentially saturated. It is shown that this condition is met exactly when the irreducible components of the restricted Douady spaces of all the cartesian powers of $X$ are compact. Some implications of saturation on Kähler-type spaces, which by a theorem of Fujiki meet the above condition, are discussed. In particular, one obatins a model-theoretic proof of the fact that relative algebraic reductions exist in the class of Kähler-type spaces.
\end{abstract}

\section{INTRODUCTION}

Recall that a subset of a complex analytic space, $A \subseteq X$, is analytic if for all $x \in X$ there are an open neighbourhood $U$ of $x$, and finitely many holomorphic functions on $U, f_{1}, \ldots, f_{m}$, such that $A \cap U$ is the set of common zeros of $f_{1}, \ldots, f_{m}$. Here is the starting point for the model theory of compact complex manifolds.

Fact 1.1. Suppose $X$ is a reduced and irreducible compact complex analytic space. Let $\mathcal{A}(X)$ be the structure whose universe is $X$ and where there is a predicate for each analytic subset of each cartesian power of $X$. Then $\mathcal{A}(X)$ satisfies:

(a) Quantifier elimination. Every definable set is a finite boolean combination of analytic sets (Łojasiewicz [20], Zilber [24]).

(b) $\omega_{1}$-Compactness. The intersection of any countable collection of definable sets is non-empty as long as every finite subcollection has non-empty intersection (Zilber [24]).

(c) Finite Morley rank. Every definable set has finite Morley rank; the Morley rank of an analytic set is bounded by its complex dimension (Zilber [24]).

As every point is analytic, $\mathcal{A}(X)$ is not $\omega$-saturated whenever $X$ is infinite; that is, there will always be collections of formulae over finitely many parameters that are finitely realisable in $\mathcal{A}(X)$ but not simultaneously realised. On the other hand, an $\omega_{1}$-compact structure in a countable language is $\omega_{1}$-saturated. One is lead to ask whether in some cases the lack of saturation is only a syntactic deficiency. This is indeed so for complex projective spaces: a consequence of Chow's theorem (and quantifier elimination) is that every set definable in $\mathcal{A}\left(\mathbb{P}_{m}(\mathbb{C})\right.$ ) is already definable with parameters in the reduct where there are only predicates for algebraic subvarieties over $\mathbb{Q}$. The latter structure is saturated.

In this paper we introduce a condition on compact complex analytic spaces, that of being essentially saturated (Section 2), which captures the situation described

Date: 29 April 2003, revised 20 September 2004.

2000 Mathematics Subject Classification. Primary 03C99; Secondary 32J99. 
above; namely, when it is a redundancy in the language of analytic sets that is responsible for the failure of saturation. Essential saturation is quite naturally related to the universal flat family of compact analytic subsets of a complex analytic space, constructed by Douady [6]. We show that our condition is met by $X$ if and only if the irreducible components of the restricted Douady spaces of $X^{n}$, for each $n>0$, are compact (Section 3). In particular, by a theorem of Fujiki [8], Kählertype spaces are essentially saturated. This class of compact complex analytic spaces is susceptible to many of the methods of algebraic geometry and the problem of their bimeromorphic classification has been studied extensively. These methods have a strong model-theoretic flavour (especially [11]), and it seems to us that essential saturation is partially responsible for this. Some model theory of Kähler-type spaces is discussed in Section 4, where we show that the class of all such spaces, viewed as a (many-sorted) structure in a canonical language given by the Douady spaces, eliminates imaginaries. As a consequence of the uniformity of definable families given by essential saturation, we obtain a model-theoretic proof of a theorem of Campana [4] and Fujiki [12]: relative algebraic reductions for fibre spaces in the class of Kähler-type spaces exist.

We have also taken this opportunity to include two related results in the appendix. The first is an observation of Anand Pillay's, that has not appeared in print (and that we require in order to avoid assuming the Continuum Hypothesis), which gives a sufficient condition for saturation in finite $U$-rank theories. Secondly, we provide the details to Anand Pillay's sketch from [22] describing how elimination of imaginaries for the class of all compact complex analytic spaces, viewed as a (manysorted) structure in the language of analytic sets, should follow from a theorem of Grauert's on meromorphic equivalence relations [14].

Here are some conventions we will adhere to in this paper:

By a complex variety we mean a reduced and irreducible complex analytic space. ${ }^{1}$

If $X$ is a reduced complex analytic space, an analytic set $A \subseteq X$ is viewed as a reduced analytic space in its own right. By the Zariski topology on $X$ we mean the topology whose closed sets are the analytic sets. If $X$ is compact then every analytic set has only finitely many irreducible components and the Zariski topology is noetherian.

A holomorphic map between compact complex varieties $f: X \rightarrow Y$ is a modification if there exist proper analytic subsets $A \subseteq X$ and $B \subseteq Y$ such that $f$ restricts to a biholomorphic map from $(X \backslash A)$ to $(Y \backslash B)$. By a meromorphic map from $X$ to $Y$ we will mean a multivalued map (written $g: X \rightarrow Y$ ) whose graph, $\Gamma(g) \subseteq X \times Y$, is an irreducible analytic set such that the first co-ordinate projection restricts to a modification $\Gamma(g) \rightarrow X$. Off a proper analytic set $g$ is a well-defined holomorphic map to $Y$. A meromorphic map $g: X \rightarrow Y$ is surjective if the second co-ordinate projection restricts to a surjection $\Gamma(g) \rightarrow Y$; and it is bimeromorphic if $\Gamma(g) \rightarrow Y$ is also a modification.

We use $\mathbb{P}_{m}(\mathbb{C})$ (or just $\mathbb{P}_{m}$ ) for projective $m$-space over the complex field, and $\mathbb{P}(\mathbb{C})^{m}$ (or just $\mathbb{P}^{m}$ ) for the $m$ th cartesian power of the projective line.

Definable means definable with parameters.

\footnotetext{
${ }^{1}$ While this may not be standard, we are following Fujiki [11] here.
} 
We suggest [7] for a modern introduction to the theory of complex analytic spaces. An introduction to, and survey of, the model theory of compact complex varieties can be found in [18], where some of the results included here were announced.

This paper stems from a chapter of my $\mathrm{PhD}$ thesis [17] written for the University of Illinois at Urbana-Champaign. I am grateful to Anand Pillay for his invaluable supervision of my graduate studies, as well as for his comments on an earlier draft of this paper. I also thank Dan Grayson for his helpful comments and suggestions.

\section{Essential Saturation}

As it is convenient to deal with all compact complex varieties at once, we will consider the many-sorted structure $\mathcal{A}$ where there is a sort for each compact complex variety, and where the language, $\mathcal{L}$, consists of a predicate for each analytic subset of a finite cartesian product of sorts. Not that here $\mathcal{L}$ and $\mathcal{A}$ are proper classes, even though the usual convention in model theory is that structures and languages are sets. The reader who is uncomfortable with this can remedy the situation by admitting as sorts only one compact complex variety from each biholomorphic equivalence class of compact complex varieties.

If $X$ is a compact complex variety, then $\mathcal{A}(X)$ can be viewed as a reduct of $\mathcal{A}$ one throws away all sorts in $\mathcal{A}$ except for $X$, and all predicates in $\mathcal{L}$ that refer to sorts other than $X$. Note that $F \subseteq X^{n}$ is definable in $\mathcal{A}$ if and only if it is definable in $\mathcal{A}(X)$ (by quantifier elimination for example).

Definition 2.1. Let $X$ be a compact complex variety. A full countable language for $X$ is a countable sublanguage, $\mathcal{L}_{0} \subset \mathcal{L}$, such that for all $n>0$, every analytic subset of $X^{n}$ is definable in $\mathcal{A}$ by an $\mathcal{L}_{0}$-formula with parameters from $\mathcal{A}$. We say that $X$ admits a full countable language if there is a full countable language for $X$.

Remark 2.2. For us "definable" always means "definable with parameters". While this is vacuous for $\mathcal{L}$-formulae (every potential parameter is itself $\emptyset$-definable), it does play a role in the above definition. Namely, the predicates in $\mathcal{L}_{0}$ are permitted to refer to other sorts, and the subsets of $X^{n}$ defined by $\mathcal{L}_{0}$-formulae may involve parameters from sorts other than $X$ itself.

Despite the cautionary tone of the above remark, the following proposition ensures the intrinsic nature of Definition 2.1.

Proposition 2.3. Let $X$ be a compact complex variety. The following conditions are equivalent:

(a) $X$ admits a full countable language.

(b) There exists a structure in a countable language, $\mathcal{M}$, with underlying universe $X$, such that for all $n>0$ and $F \subseteq X^{n}, F$ is definable in $\mathcal{A}(X)$ if and only if it is definable in $\mathcal{M}$.

(c) There is a full countable language for $X, \mathcal{L}_{0}$, such that each predicate in $\mathcal{L}_{0}$ corresponds to an analytic subset of a finite cartesian power of $X$.

Moreover, if this is the case and $\mathcal{M}$ is as in (b), then $\mathcal{M}$ is saturated. ${ }^{2}$

\footnotetext{
${ }^{2} \mathrm{~A}$ structure is "saturated" if it is $\kappa$-saturated for $\kappa$ the cardinality of the universe.
} 
Proof. First assume that $\mathcal{L}_{0}$ is a full countable language for $X$. Let $\mathcal{A}_{0}$ be the reduct of $\mathcal{A}$ to an $\mathcal{L}_{0}$-structure, consisting of the sort $X$ together with all the other sorts appearing in $\mathcal{L}_{0}$. Note that by finiteness of Morley rank, $\operatorname{Th}\left(\mathcal{A}_{0}\right)$ is stable.

Let $\phi(x, z)$ be any $\mathcal{L}_{0}$-formula where $x$ is a tuple of variables belonging to the sort $X$ and $z$ is a tuple of variables belonging to a cartesian product of sorts $Z$. By uniform definablility of types in stable theories (see III.1.24 [1]), there exists an $\mathcal{L}_{0}$-formula $d_{\phi}(x, y)$, where $y$ is now a tuple of variables also belonging to the sort $X$, with the following property: for all $b \in Z$ there exists $c$ from $X$ such that $d_{\phi}(x, c)$ is the $\phi$-definition of the $\mathcal{L}_{0}$-type of $b$ over $X$ in $\mathcal{A}_{0}$. That is, for all $a$ from $X, \mathcal{A}_{0} \models d_{\phi}(a, c)$ iff $\mathcal{A}_{0} \models \phi(a, b)$. So $d_{\phi}(x, c)$, which is an $\mathcal{L}_{0}$-formula with parameters from $X$, defines the same set as $\phi(x, b)$.

Let $\mathcal{M}$ be the structure whose universe is $X$ and where there is a predicate for each $d_{\phi}(x, y)$ obtained in the above manner. The language of $\mathcal{M}$ is countable (as $\mathcal{L}_{0}$ is countable). It follows from the construction that a subset of $X^{n}$ is definable in $\mathcal{M}$ if and only if it is definable in $\mathcal{A}_{0}$. As $\mathcal{L}_{0}$ is a full countable language for $X$, and as $\mathcal{A}$ admits quantifier elimination, definability in $\mathcal{A}_{0}$ is in turn equivalent to definability in $\mathcal{A}(X)$. We have shown that $(a)$ implies $(b)$.

For $(b)$ implies $(c)$, note that there are only countably many $\emptyset$-definable sets in $\mathcal{M}$, each of which, being definable in $\mathcal{A}(X)$, is a finite boolean combination of analytic subsets of cartesian powers of $X$. Let $\mathcal{L}_{0}:=\left\{P_{A_{i}}: i \in I\right\}$ be the countable collection of predicates corresponding to these analytic sets. Then $\mathcal{L}_{0}$ is a full countable language for $X$ satisfying $(c)$.

That $(c)$ implies $(a)$ is immediate.

Finally, suppose that $X$ does admit a full countable language and that $\mathcal{M}$ is as in part (b) of the propostion. Then $\mathcal{M}$ is $\omega_{1}$-compact (as $\mathcal{A}(X)$ is) in a countable language, and hence is $\omega_{1}$-saturated. As the cardinality of $X$ is the continuum (assuming $\operatorname{dim} X>0$ ), what we actually need to show is that $\mathcal{M}$ is $2^{\omega}$-saturated. By Proposition 5.1 and Corollary 5.4 of the appendix, it suffices to show that every nonalgebraic type over countably many parameters has continuum many realisations in $\mathcal{M}$. Suppose $p$ is a non-algebraic $n$-type over countably many parameters. By the countability of the language of $\mathcal{M}$ there are only countably many formulae in $p$. By quantifier elimination in $\mathcal{A}(X)$, and the non-algebraicity of $p$, there is an irreducible analytic set $A \subseteq X^{n}$ of positive dimension, such that $p$ is realised in $\mathcal{M}$ by a countable intersection of non-empty Zariski open subsets of $A$. It follows, using the fact that a proper analytic subset of $A$ is nowhere dense, that the cardinality of the set of realisations of $p$ is the continuum.

Proposition 2.3 justifies the following terminology: we will say that a compact complex variety $X$ is essentially saturated to mean that the equivalent conditions of Proposition 2.3 are satisfied.

Example 2.4. Consider $\mathbb{P}_{m}(\mathbb{C})$. As mentioned in the introduction, if we take $\mathcal{L}_{0}$ to be those predicates that correspond to algebraic subvarieties of cartesian powers of $\mathbb{P}_{m}$ over $\mathbb{Q}$, then $\mathcal{L}_{0}$ is a full countable language for $\mathcal{A}\left(\mathbb{P}_{m}\right)$. The class of essentially saturated compact complex varieties is clearly preserved under taking subvarieties, and hence all projective varieties are essentially saturated. We will obtain many more examples (and non-examples) in the next section, where we explore the connection between full countable languages and the compactness of Douady spaces. 
We do not know whether cartesian products of essentially saturated compact complex varieties are again essentially saturated. Nevertheless, we may extend Definition 2.1 as follows: A pair of compact complex varieties $\{X, Y\}$ is said to be essentially saturated if for every $n, m>0, X^{n} \times Y^{m}$ is essentially saturated. Just as in Propostion 2.3, the following are equivalent:

- $\{X, Y\}$ is essentially saturated.

- There is a countable sublanguage, $\mathcal{L}_{0} \subset \mathcal{L}$, such that every analytic subset of $X^{n} \times Y^{m}$ is definable in $\mathcal{A}$ by an $\mathcal{L}_{0}$-formula with parameters from $\mathcal{A}$.

- There is a 2 -sorted structure $\mathcal{M}$, in a countable language, whose sorts are $\{X, Y\}$, and such that a subset of $X^{n} \times Y^{m}$ is definable in $\mathcal{M}$ if and only if it is definable in $\mathcal{A}$.

Again, such an $\mathcal{M}$ will be a saturated structure. There is nothing special about "two" here; one can do the same thing with any finite (or even countable) number of compact complex varieties.

We end this section by pointing out that if we begin with an essentially saturated compact complex variety, then we can add any projective variety as a sort, and remain essentially saturated. In fact, we will restrict our attention to the projective line $\mathbb{P}(\mathbb{C})$ (which suffices). But first a lemma:

Lemma 2.5. Suppose $X$ is an essentially saturated compact complex variety, $D$ is a definable subset of some cartesian power of $X$, and $g: D \rightarrow Y$ is a surjective definable map onto a compact complex variety. Then $\{X, Y\}$ is essentially saturated.

Proof. Let $\mathcal{M}$ be a structure with universe $X$, in a countable language, that witnesses the essential saturation of $X$. Let $\mathcal{M}_{Y}$ be the structure obtained from $\mathcal{M}$ by adding a sort for $Y$, as well as a predicate for the graph of $g$ (note that $D$ is already definable in $\mathcal{M}$ ). The language of $\mathcal{M}_{Y}$ is still countable, and clearly every set definable in $\mathcal{M}_{Y}$ is definable in $\mathcal{A}$. On the other hand, suppose $F \subseteq X^{n} \times Y^{m}$ is definable in $\mathcal{A}$. Let $h: X^{n} \times D^{m} \rightarrow X^{n} \times Y^{m}$ be the surjection id $\times g^{m}$. Then $h$ is definable in $\mathcal{M}_{Y}$. Now, $h^{-1}(F) \subseteq X^{n} \times D^{m}$, is definable in $\mathcal{A}$, and hence in $\mathcal{M}$, and hence in $\mathcal{M}_{Y}$. But then, $F=h\left(h^{-1}(F)\right)$ is definable in $\mathcal{M}_{Y}$. We have shown that $\mathcal{M}_{Y}$ witnesses the essential saturation of $\{X, Y\}$.

Proposition 2.6. If $X$ is essentially saturated then so is $\{X, \mathbb{P}(\mathbb{C})\}$.

Proof. Suppose that for all $n, m>0$ and analytic $A \subseteq X^{n} \times \mathbb{P}^{m}, A$ is the product of an analytic set in $X^{n}$ and an analytic set in $\mathbb{P}^{m}$. Then we obtain the desired full countable language for $X^{n} \times \mathbb{P}^{m}$ by taking the union of the full countable languages for $X$ and $\mathbb{P}$. Hence, we may assume that for some $n, m>0$, there is a subvariety $A \subset X^{n} \times \mathbb{P}^{m}$ that is not the product of analytic sets in $X^{n}$ and $\mathbb{P}^{m}$. We show that $\mathbb{P}$ is then the definable image of a subset of $X^{n}$. By Lemma 2.5, this will suffice.

Let $p(x, y)$ be the generic type of $A$; the type saying that $(x, y)$ is in $A$ but not in any proper subvariety. This type is consistent by the noetherianity of the Zariski topology and complete by quantifier elimination. Let $(a, b) \models p$ be a realisation in some elementary extension, $\mathcal{A}^{\prime}$ of $\mathcal{A} .^{3}$ Note that $\operatorname{Th}(\mathcal{A}(\mathbb{P}))$ is strongly minimal and admits elimination of imaginaries (it is definably bi-interpretable with $\operatorname{Th}(\mathbb{C},+, \times, 0,1))$. Our assumption on $A$ implies that $b$ forks with $a$; the Morley rank

${ }^{3} \mathrm{~A}$ discussion of elementary extensions of $\mathcal{A}$ and how they relate to the geometry of $\mathcal{A}$ itself can be found in [19]. 
of $\operatorname{tp}(b / a)$ is strictly less than that of $\operatorname{tp}(b)$. We obtain a generic tuple $\left(b_{1}, \ldots, b_{r}\right)$ of $\mathbb{P}^{r}$ in $\mathcal{A}^{\prime}$, such that $b_{r} \in \operatorname{acl}\left(b_{1}, \ldots, b_{r-1}, a\right)$. Elimination of imaginaries for $\operatorname{Th}(\mathcal{A}(\mathbb{P}))$ allows us to replace $b_{r}$ with an element $c$ in $\mathcal{A}^{\prime}$ such that $\left(b_{1}, \ldots, b_{r-1}, c\right)$ is still a generic of $\mathbb{P}^{r}$, but now $c \in \operatorname{dcl}\left(b_{1}, \ldots, b_{r-1}, a\right)$. Let $\bar{b}:=\left(b_{1}, \ldots, b_{r-1}\right)$, and let $f_{\bar{b}}: X^{n}\left(\mathcal{A}^{\prime}\right) \rightarrow \mathbb{P}(\mathbb{C})\left(\mathcal{A}^{\prime}\right)$ be a partial $\bar{b}$-definable map witnessing $c \in \operatorname{dcl}(\bar{b}, a)$. The genericity of $(\bar{b}, c)$ in $\mathbb{P}^{r}$, and strong minimality, implies that there is a subset $D_{\bar{b}} \subseteq X^{n}\left(\mathcal{A}^{\prime}\right)$, definable over $\bar{b}$, such that $f_{\bar{b}}\left(D_{\bar{b}}\right)$ is cofinite in $\mathbb{P}(\mathbb{C})\left(\mathcal{A}^{\prime}\right)$. By transfer back to the standard model, some cofinite subset of $\mathbb{P}(\mathbb{C})$ is the definable image of a definable subset of $X^{n}$. Hence all of $\mathbb{P}(\mathbb{C})$ is the definable image of a subset of $X^{n}$, as desired.

\section{Douady Spaces}

Let us introduce some conventions regarding the fibres of a holomorphic map. Suppose $f: G \rightarrow S$ is a surjective holomorphic map between complex analytic spaces. At the level of points we can view this as a family of analytic subsets of $G$ parametrised by $S$ : each point $s \in S$ gives rise to the analytic set $G_{s}:=f^{-1}(s) \subseteq G$. If $G$ is given as an analytic subspace of $S \times X$, for some other complex analytic space $X$, and $f$ is the co-ordinate projection, then we often identify $G_{s}$ with the corresponding analytic set in $X$, namely $\{x \in X:(s, x) \in G\}$ - thereby viewing $G$ as a family of analytic subsets of $X$ parametrised by $S$. On the other hand, we can view $f: G \rightarrow S$ as a family of analytic subspaces of $G$ : each $s \in S$ gives rise to a possibly non-reduced complex analytic space, $\{s\} \times{ }_{S} G$, whose underlying set can be identified with $G_{s}$. We will refer to the analytic space $\{s\} \times_{S} G$ as the fibre (or the sheaf-theoretic fibre); and to the analytic set $G_{s}$ as the set-theoretic fibre. Note that even when $G$ and $S$ are reduced, it is not necessarily the case that all the fibres will be reduced; that is, the sheaf-theoretic fibres and the set-theoretic fibres may not coincide. For example, if $G$ is the subvariety of $\mathbb{C} \times \mathbb{C}^{2}$ given by $x^{2}-u y=0$ in the co-ordinate variables $(u, x, y)$, then the fibre of $G$ above $u=0$ is a double line in $\mathbb{C}^{2}$; it is not reduced.

We recall the universal family for compact analytic subspaces of a given complex analytic space, constructed by Douady in [6]. Let $X$ be any complex analytic space (possibly non-compact and non-reduced). Then there exists a complex analytic space $\mathcal{D}=\mathcal{D}(X)$ and an analytic subspace $\mathcal{Z}=\mathcal{Z}(X) \subseteq \mathcal{D} \times X$ such that:

(a) The projection $\mathcal{Z} \rightarrow \mathcal{D}$ is a flat and proper surjection.

(b) If $S$ is a complex analytic space and $G$ is an analytic subspace of $S \times X$ that is flat and proper over $S$, then there exists a unique holomorphic map $g: S \rightarrow \mathcal{D}$ such that $G \simeq S \times_{\mathcal{D}} \mathcal{Z}$ canonically.

$\mathcal{D}(X)$ is called the Douady Space of $X, \mathcal{Z}(X)$ is called the universal family of $X$, and $g: S \rightarrow \mathcal{D}(X)$ as in (b) is called the Douady map associated to $G \rightarrow S$. An important fact, due to Fujiki [9], is that $\mathcal{D}(X)$ has only countably many irreducible components. In the case that $X$ is a projective variety, $\mathcal{D}(X)$ is the Hilbert scheme of $X$. A more detailed discussion of Douady spaces can be found in [5].

By way of explanation let us point that the compact analytic subsets of $X$ are in bijective correspondence with the points in $\mathcal{D}(X)$ above which the fibre of $\mathcal{Z}(X) \rightarrow$ $\mathcal{D}(X)$ is reduced. Indeed, if $A \subseteq X$ is a compact analytic set, then applying (b) to $S:=\{s\}$ a 0 -dimensional variety and $G:=S \times A$, gives us a holomorphic 
map $g:\{s\} \rightarrow \mathcal{D}$ such that $A$ "is" the fibre of $\mathcal{Z} \rightarrow \mathcal{D}$ above $g(s)$. More precisely, $\{s\} \times_{\mathcal{D}} \mathcal{Z}$ (which is the sheaf-theoretic fibre of $\mathcal{Z} \rightarrow \mathcal{D}$ over $g(s)$ ) is isomorphic, under the projection $\{s\} \times_{\mathcal{D}} \mathcal{Z} \rightarrow X$, to $A$. As $A$ is reduced, so is the fibre. In fact, by the uniqueness of the Douady map, $g(s)$ is the unique point in $\mathcal{D}$ such that: (1) the fibre of $\mathcal{Z} \rightarrow \mathcal{D}$ above $g(s)$ is reduced, and (2) $A=\{a \in X:(g(s), a) \in \mathcal{Z}\}=\mathcal{Z}_{g(s)}$. We sometimes denote $g(s)$ by $[A]$, and refer to it as the Douady point of $A$. That this injective association is bijective follows from the observation that for any $d \in \mathcal{D}$, $\mathcal{Z}_{d}$ is compact (this is the properness of $\mathcal{Z} \rightarrow \mathcal{D}$ ) and if $\{d\} \times_{\mathcal{D}} \mathcal{Z}$ is reduced then $d=\left[\mathcal{Z}_{d}\right]$

We have been considering the reduced fibres of $\mathcal{Z}(X) \rightarrow \mathcal{D}(X)$. The non-reduced fibres correspond to compact subspaces of $X$ equipped with non-reduced structure sheaves. These cannot be entirely avoided (even when $X$ is assumed to be reduced and compact). Nevertheless, following Fujiki [8], we can avoid "most" of them by restricting our attention to a certain subspace of the Douady space:

First recall that for any complex analytic space $X$, the reduction of $X, X_{\text {red }}$, is the reduced complex analytic subspace of $X$ obtained by quotienting out the structure sheaf of $X$ by its nilradical ideal sheaf.

Definition 3.1. Suppose $X$ is a compact complex variety. Let $D(X)$ be the subspace of $\mathcal{D}(X)$ that is obtained by taking the union of all those irreducible components of $\mathcal{D}(X)_{\text {red }}, D_{\alpha}$, such that for some $d \in D_{\alpha}$, the (sheaf-theoretic) fibre of $\mathcal{Z}(X)$ over $d$ is reduced and pure-dimensional (i.e. the irreducible components of $\mathcal{Z}(X)_{d}$ have the same dimension). Let $Z(X) \subseteq D(X) \times X$ be the corresponding subspace of $\mathcal{Z}(X)$ over $D(X)$. We call $D(X)$ the restricted Douady space of $X$, and $Z(X)$ the restricted universal family of $X$.

Remark 3.2. The above definition is justified by the following observations. Suppose $f: G \rightarrow S$ is a proper and flat holomorphic surjection between complex analytic spaces where $S$ is reduced and irreducible. A theorem of Bănică [2] tells us that if $G$ is reduced then the set of points $s \in S$ such that the (sheaf-theoretic) fibre of $G$ above $s$ is reduced, is a non-empty Zariski open subset of $S$. Conversely, if even one fibre of $f$ is reduced and pure-dimensional, then $G$ is also reduced and pure-dimensional (see Lemma 3 of [10]). Flatness then implies that every fibre of $G \rightarrow S$ is pure-dimensional (and of the same dimension). Now suppose $D_{\alpha}$ is an irreducible component of $\mathcal{D}(X)_{\text {red }}$ and let $Z_{\alpha}:=D_{\alpha} \times_{\mathcal{D}(X)} \mathcal{Z}(X) \subseteq D_{\alpha} \times X$ be the corresponding subspace of the universal family over $D_{\alpha}$. The facts just discussed imply that the following are equivalent:

- $D_{\alpha}$ is a component of the restricted Douady space, $D(X)$,

- $Z_{\alpha}$ is reduced and pure-dimensional,

- All the fibres of $Z_{\alpha} \rightarrow D_{\alpha}$ are pure-dimensional of a fixed dimension, and the set of points $d \in D_{\alpha}$ such that the (sheaf-theoretic) fibre of $Z_{\alpha}$ above $d$ is reduced is a non-empty Zariski open subset of $D_{\alpha}$.

The restricted Douady space is sufficiently rich for our purposes; the collection of pure-dimensional compact analytic subsets of $X$ are in bijective correspondence with a dense Zariski open subset of $D(X)$. Moreover, if $S$ is a complex variety and $G \subseteq S \times X$ is a pure-dimensional analytic subset that is flat and proper over $S$, then some fibre of $G \rightarrow S$ is reduced and pure-dimensional and so the Douady map takes $S$ to an irreducible component $D_{\alpha}$ of $D(X)$. At the level of points, this 
means that $g$ extends by identity to a map from $G$ to $Z_{\alpha}$,

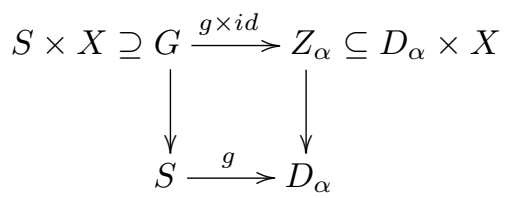

such that $G_{s}=\left(Z_{\alpha}\right)_{g(s)}$ for all $s \in S$. Roughly speaking, every flat family of pure-dimensional analytic subsets of $X$ lives in $Z(X) \rightarrow D(X)$.

We now use Douady spaces to characterise those compact complex varieties that admit a full countable language.

Theorem 3.3. A compact complex variety $X$ is essentially saturated if and only if for all $n>0$, every irreducible component of $D\left(X^{n}\right)$ is compact.

Proof. Suppose each irreducible component of $D\left(X^{n}\right)$ is compact, and fix such a component $D_{\alpha}$. Then $D_{\alpha}$ is a sort in $\mathcal{A}$. Let $Z_{\alpha}$ be the universal family of $X^{n}$ over $D_{\alpha}$. Then $Z_{\alpha}$ is an analytic subset of $D_{\alpha} \times X^{n}$, and hence a named predicate in $\mathcal{L}$. Let $\mathcal{L}_{0}$ be the sublanguage of $\mathcal{L}$ consisting of all relation symbols corresponding to such $Z_{\alpha}$, as $\alpha$ and $n$ vary. Since $D\left(X^{n}\right)$ has only countably many components for each $n, \mathcal{L}_{0}$ is countable. Every pure-dimensional analytic subset of $X^{n}$ occurs as a fibre of $Z\left(X^{n}\right) \rightarrow D\left(X^{n}\right)$. In particular, if $A \subseteq X^{n}$ is an irreducible analytic set then the Douady point of $A, d:=[A]$, lives in some $D_{\alpha}$ and $A=\left(Z_{\alpha}\right)_{d}$. Hence $A$ is $\mathcal{L}_{0}$-definable (over $d$ ). It follows that every analytic set is $\mathcal{L}_{0}$-definable, and so $X$ admits a full countable language.

To prove the converse we need to understand families of analytic sets that are definable in $\mathcal{A}$. By a definable family of analytic subsets of $X^{n}$ in $\mathcal{A}$ we mean a subset $F \subseteq B \times X^{n}$ where $F$ and $B$ are definable in $\mathcal{A}$, the co-ordinate projection $F \rightarrow B$ is surjective, and $F_{b}:=\left\{x \in X^{n}:(b, x) \in F\right\}$ is an analytic subset of $X^{n}$ for each $b \in B$. Such a family is analytic, if $B$ is locally Zariski closed and irreducible (i.e., $B$ is a Zariski open subset of an irreducible analytic subset of some cartesian product of sorts from $\mathcal{A}$ ), and $F$ is (relatively) Zariski closed in $B \times X^{n}$. Note that in this case $F$ and $B$ inherit the structure of reduced complex analytic spaces and $F \rightarrow B$ is a proper holomorphic surjection. An analytic family is flat if $F \rightarrow B$ is flat.

Lemma 3.4. Suppose $X$ is an essentially saturated compact complex variety. For each $n>0$, there are countably many definable flat analytic families of analytic subsets of $X^{n}$ in $\mathcal{A},\left(F_{i} \rightarrow B_{i}\right)_{i \in \omega}$ such that:

(i) for each $i$, every fibre of $F_{i} \rightarrow B_{i}$ is reduced,

(ii) every analytic subset of $X^{n}$ occurs as a fibre of some $F_{i} \rightarrow B_{i}$, and,

(iii) for each $i$, the Douady map associated to $F_{i} \rightarrow B_{i}, g_{i}: B_{i} \rightarrow \mathcal{D}\left(X^{n}\right)$, is definable in $\mathcal{A}$. That is, if $\overline{B_{i}}$ denotes the Zariski closure of $B_{i}$ and $\overline{g\left(B_{i}\right)}$ denotes the Zariski closure of $g\left(B_{i}\right)$ in $\mathcal{D}\left(X^{n}\right)$, then $\overline{g\left(B_{i}\right)}$ is compact and $g_{i}$ extends to a meromorphic map $\overline{B_{i}} \rightarrow \overline{g_{i}\left(B_{i}\right)}$.

Proof. The existence of a full countable language for $X$, together with quantifier elimination, gives us, for each $n>0$, countably many definable analytic families of analytic subsets of $X^{n}$ such that every analytic set appears as the set-theoretic fibre of at least one of them. It suffices therefore, to show that for any such family $F \rightarrow B$ there is a definable decomposition, $B=B_{1} \cup \cdots \cup B_{m}$, such that the 
restriction of $F$ to each $B_{j}, F_{B_{j}}:=F \cap\left(B_{j} \times X\right) \rightarrow B_{j}$, is analytic, flat, has all reduced fibres, and satisfies the condition in part (iii) of the lemma (the associated Douady map is definable). By induction on $\operatorname{dim} B$ this in turn reduces to showing that there exists a non-empty Zariski open subset $U \subseteq B$ such that $F_{U} \rightarrow U$ has only reduced fibres, is flat, and satisfies (iii).

As $F \rightarrow B$ is an analytic family, $B$ is a Zariski open subset of a compact complex variety $\bar{B}$, and $F$ is the intersection of an analytic set $\bar{F} \subseteq \bar{B} \times X^{n}$ with $B \times X^{n}$. Consider $\bar{F} \rightarrow \bar{B}$, which is a holomorphic surjection between compact complex varieties. Hironaka's flattening theorem [15] provides a bimeromorphic copy of $\bar{F} \rightarrow \bar{B}$ that is flat: there is a modification of compact complex varieties $h: \widehat{B} \rightarrow \bar{B}$ and an irreducible analytic set $\widehat{F}$ in $\widehat{B} \times \bar{B} \bar{F}$ such that $\widehat{F} \rightarrow \widehat{B}$ is flat, and such that $\widehat{F}_{b}=\bar{F}_{h(b)}$ for all $b$ in some non-empty Zariski open subset of $\widehat{B}$. In diagrams,

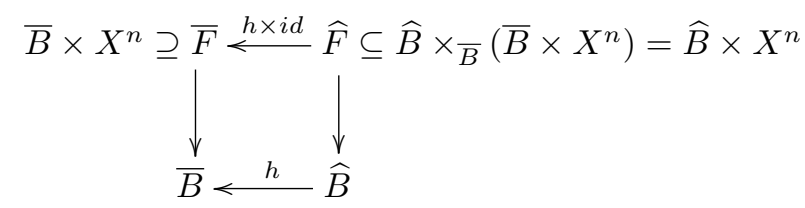

As $\widehat{F} \rightarrow \widehat{B}$ is flat and $\widehat{F}$ is reduced, there is a non-empty Zariski open subset $V \subseteq \widehat{B}$ over which the fibres of $\widehat{F} \rightarrow \widehat{B}$ are reduced (Bănică [2]). Shrinking $V$ further, we may assume that $h$ restricted to $V$ is an isomorphism, its image is a non-empty Zariski open set $U \subseteq B$, and $\widehat{F}_{b}=\bar{F}_{h(b)}$ for all $b \in V$. Hence $\widehat{F}_{V} \rightarrow V$ is isomorphic to $F_{U} \rightarrow U$ over $h$. So $F_{U} \rightarrow U$ is flat and all its fibres are reduced. Moreover, there is a Douady map $\widehat{B} \rightarrow \mathcal{D}\left(X^{n}\right)$ associated to $\widehat{F} \rightarrow \widehat{B}$. If we compose the meromorphic map $h^{-1}: \bar{B} \rightarrow \widehat{B}$ with this Douady map $\widehat{B} \rightarrow \mathcal{D}\left(X^{n}\right)$, we obtain a meromorphic map $g: \bar{B} \rightarrow \mathcal{D}\left(X^{n}\right)$. The uniqueness of Douady maps ensures that $g$ restricted to $U$ is the Douady map associated to $F_{U} \rightarrow U$. We have shown that $F_{U} \rightarrow U$ satisfies condition (iii): the Zariski closure of $g(U)$ in $\mathcal{D}\left(X^{n}\right)$ is compact (it is the image of the compact $\widehat{B}$ ) and $g: \bar{B} \rightarrow \overline{g(U)}$ witnesses that the Douady map associated to $F_{U} \rightarrow U$ is definable in $\mathcal{A}$.

Now for the remaining direction of Theorem 3.3. Suppose $X$ is an essentially saturated compact complex variety. Fix $n>0$. We need to show that every irreducible component of $D\left(X^{n}\right)$ is compact. Let $\left(F_{i} \rightarrow B_{i}\right)_{i \in \omega}$ be the collection of those families given by Lemma 3.4 that have some pure-dimensional fibre. As that fibre is also reduced (part of the lemma) the Douady map actually takes each $B_{i}$ to $D\left(X^{n}\right), g_{i}: B_{i} \rightarrow D\left(X^{n}\right)$. Moreover, as every pure-dimensional analytic subset of $X^{n}$ must appear as a (reduced) fibre in one of these families, $\bigcup_{i \in \omega} g_{i}\left(B_{i}\right)$ must cover those points in $D\left(X^{n}\right)$ over which $Z\left(X^{n}\right)$ has a reduced fibre. Fix an irreducible component, $D_{\alpha}$, of $D\left(X^{n}\right)$. There is a proper analytic set $E \subset D_{\alpha}$ such that the fibres above $D_{\alpha} \backslash E$ are reduced (see Remark 3.2). Hence, $D_{\alpha} \backslash E \subseteq \bigcup_{i \in \omega} g_{i}\left(B_{i}\right)$ and so $D_{\alpha}=E \cup \bigcup_{i \in \omega}\left(\overline{g_{i}\left(B_{i}\right)} \cap D_{\alpha}\right)$. A consequence of Baire Category is that no complex variety can be written as the countable union of proper analytic subsets. Hence $D_{\alpha}=\overline{g_{i}\left(B_{i}\right)} \cap D_{\alpha}$ for some $i \in \omega$. In fact, as $\overline{g_{i}\left(B_{i}\right)} \subset D\left(X^{n}\right)$ is irreducible it must 
be that $D_{\alpha}=\overline{g_{i}\left(B_{i}\right)}$. In any case, $\overline{g_{i}\left(B_{i}\right)}$ is compact by Lemma 3.4 , and so $D_{\alpha}$ is compact. This completes the proof of Theorem 3.3.

Example 3.5. (Hopf surfaces) We obtain an example of a compact complex variety that is not essentially saturated. Fix $\alpha$ a real number strictly between 0 and 1, and let $\Gamma \leq G L(2, \mathbb{C})$ be the cyclic subgroup generated by $\left(\begin{array}{ll}\alpha & 0 \\ 0 & \alpha\end{array}\right)$. Let $W$ be the analytic space $\mathbb{C}^{2} \backslash\{(0,0)\}$. We can view $\Gamma$ as a group of analytic isomorphisms of $W$ generated by $\left(z_{1}, z_{2}\right) \mapsto\left(\alpha z_{1}, \alpha z_{2}\right)$. Then $H:=W / \Gamma$, the quotient of $W$ by the action of $\Gamma$, is a two-dimensional compact complex manifold called a Hopf surface. Fujiki has pointed out that $D(H \times H)$ has a non-compact component. We sketch the argument here as it is rather instructive (see [5]). Identify $\operatorname{Aut}(H)$ with the set of points $g \in D\left(H^{2}\right)$ such that $Z\left(H^{2}\right)_{g}$ is the graph of an analytic automorphism of $H$. This makes Aut $(H)$ into a Zariski open subset of $D\left(H^{2}\right)$ that inherits the structure of a complex Lie group (under composition). Let $G$ be the connected component of the identity in $\operatorname{Aut}(H)$. That is, $G:=\operatorname{Aut}(H) \cap C$, where $C$ is the irreducible component of $D\left(H^{2}\right)$ containing the Douady point of the diagonal in $H^{2}$. Assume that $C$ is compact, and hence $G$ is a definable group in $\mathcal{A}$. Let $S \leq G$ be the subgroup of elements that stabilise the image of both $(1,0)$ and $(0,1)$ in $H$. Then $S$ is definable. On the other hand, the automorphisms of $H$ in $G$ are exactly those that are induced by linear transformations of $\mathbb{C}^{2}$ restricted to $W$. That is, $G$ is isomorphic to $G L(2, \mathbb{C}) / \Gamma$. This identifies $S$ with the group of matrices of the form $\left(\begin{array}{cc}\alpha^{m} & 0 \\ 0 & \alpha^{n}\end{array}\right)$, modulo $\Gamma$. But by quantifier elimination, no definable subset of a compact complex variety can be countably infinite. Hence $C$ is not compact. By Theorem 3.3, a Hopf surface is not essentially saturated.

\section{KÄHLER-TYPE VARIETIES}

In this section we describe a particularly relevant class of essentially saturated compact complex varieties, and then describe an application of model-theoretic methods to this class.

Suppose $M$ is an $n$-dimensional complex manifold. Viewing $M$ as a real $2 n$ dimensional $C^{\infty}$ manifold, let $T_{p} M$ denote the real tangent space to $M$ at $p \in M$, and $J_{p}: T_{p} \rightarrow T_{p}$ the complex structure ( $J_{p}$ is an $\mathbb{R}$-linear map such that $J_{p}^{2}=\mathrm{id}$ ). Suppose $\omega \in \Omega^{2}(M)$ is a real symplectic 2-form; so for every $p \in M, \omega_{p}$ induces a non-degenerate alternating bilinear map $B_{p}: T_{p} M \times T_{p} M \rightarrow \mathbb{R}$, and $d \omega=0$. Then $\omega$ is said to be Kähler if for every $p \in M$

- $\omega_{p}$ is $J_{p}$-compatible: $B_{p}\left(J_{p} v, J_{p} w\right)=B_{p}(v, w)$, and

- $Q_{p}$ given by $Q_{p}(v, w):=B_{p}\left(v, J_{p} w\right)$ is positive definite.

A complex manifold is Kähler if there exists a Kähler 2-form on $M$. In this case $p \mapsto\left(Q_{p}+i B_{p}\right)$ defines a Hermitian metric on $M$. Indeed, a Kähler manifold can be alternatively described as a complex manifold which admits a Hermitian metric whose fundamental form of type $(1,1)$ is closed. Examples of compact Kähler manifolds include projective manifolds and complex tori.

A compact complex variety is of Kähler-type if it is the holomorphic image of a compact Kähler manifold (introduced by Fujiki in [8]). The class of Kähler-type 
varieties is preserved under meromorphic images, cartesian products, and subvarieties. The problem of classifying Kähler-type varieties has been studied extensively, largely because many of the methods of algebraic geometry extend to this class (see for example [11]). That Kähler-type varieties are also very meaningful from the model-theoretic point of view is contained in the following fact:

Fact 4.1. If $X$ is of Kähler-type then every irreducible component of $D(X)$ is compact (Fujiki [8]), and is itself of Kähler-type (Fujiki [13], Campana [3]).

In particular, every Kähler-type variety is essentially saturated.

Question 4.2. It is not known (to this author) whether every essentially saturated variety is of Kähler-type. By Theorem 3.3, this is equivalent to asking: Can Kählertype varieties be characterised as those compact complex varieties all of whose cartesian powers have restricted Douady spaces whose components are compact? Note that the reference to cartesian powers is necessary - if $H$ is a Hopf surface then $D(H)$ does have compact components but $D\left(H^{2}\right)$ does not (see Example 3.5).

Fact 4.1 tells us more than essential saturation for each individual Kähler-type variety, it allows us to work simultaneously with all Kähler-type varieties equipped with canonical full countable languages:

Definition 4.3. Let $\mathcal{C}$ be the many-sorted structure where there is a sort for each Kähler-type variety and where for each finite cartesian product of sorts, $Y$, and each irreducible component $D_{\alpha}$ of $D(Y)$ (also of Kähler-type by Fact 4.1), there is a predicate for $Z_{\alpha} \subseteq D_{\alpha} \times Y$, the corresponding subspace of the universal family over $D_{\alpha}$. We refer to this as the Douady language.

While the Douady language is not itself countable, for any given Kähler-type variety $X$, those predicates in the Douady language that refer only to $X$ - that is the components $Z_{\alpha} \rightarrow D_{\alpha}$ of $Z\left(X^{n}\right) \rightarrow D\left(X^{n}\right)$ for all $n \in \mathbb{N}$ - form a full countable language for $X$. In this way $\mathcal{C}$ provides a setting in which to work with all Kählertype varieties, equipped with full countable languages, simultaneously. That these languages are "canonical" is expressed by the universality of Douady spaces.

We will show that $\operatorname{Th}(\mathcal{C})$ eliminates imaginaries (after naming parameters). This is also true of $\operatorname{Th}(\mathcal{A})$ itself; a proof based on Pillay's suggestion is included in the appendix of this paper (Proposition 5.2 and Corollary 5.4). While elimination of imaginaries for $\operatorname{Th}(\mathcal{C})$ could also be extracted from the proof of elimination of imaginaries for $\operatorname{Th}(\mathcal{A})$ (though not just from the statement), the argument we present below is more direct and exhibits some of the advantages of working in $\mathcal{C}$.

Proposition 4.4. The elementary diagram of $\mathcal{C}$ eliminates imaginaries.

Proof. By the elementary diagram of $\mathcal{C}$ we mean $\operatorname{Th}(\mathcal{C}, s: s \in \mathcal{C})$; the theory of the structure obtained from $\mathcal{C}$ by naming every element. Suppose $X$ is a Kählertype variety. Let $\left\{X_{i}: i \in \omega\right\}$ be the smallest collection of sorts of $\mathcal{C}$ containing $X$ that is closed under cartesian products and is closed under taking irreducible components of restricted Douady spaces. Let $\left.\mathcal{C}\right|_{X}$ be the full reduct of $\mathcal{C}$ to the sorts $\left\{X_{i}: i \in \omega\right\}$. That is, for each finite cartesian product of the $X_{i}$ 's, $Y$, and each irreducible component $D_{\alpha}$ of $D(Y)$, there is a predicate for $Z_{\alpha} \subseteq D_{\alpha} \times Y$. Let $U(Y, \alpha) \subseteq D_{\alpha}$ be the set of points over which the fibres of $Z_{\alpha} \rightarrow D_{\alpha}$ are reduced. Then $U(Y, \alpha)$ is a non-empty Zariski open subset of $D_{\alpha}$ (see Remark 3.2); and hence definable in $\left.\mathcal{C}\right|_{X}$. Let $S$ be a countable set of parameters over which all such 
$U(Y, \alpha)$ (as $Y$ and $\alpha$ vary) are definable in $\left.\mathcal{C}\right|_{X}$. Ranging over all $X$, the proposition will follow from:

Lemma 4.5. $\operatorname{Th}\left(\left.\mathcal{C}\right|_{X}, s: s \in S\right)$ eliminates imaginaries.

Proof. Note that $\left(\left.\mathcal{C}\right|_{X}, s: s \in S\right)$ is a saturated structure. Indeed, it is $\omega_{1}$-compact in a countable language, and $2^{\omega}$-saturation follows by 5.1 (as in 2.3). It suffices therefore, to show that every definable set, $F$, has a code: there exists a tuple $c$ from $\left.\mathcal{C}\right|_{X}$ such that if $\sigma$ is an automorphism of $\left(\left.\mathcal{C}\right|_{X}, s: s \in S\right)$, then $\sigma(F)=F$ if and only if $\sigma(c)=c$.

Suppose $Y$ is a finite cartesian product of sorts from $\left.\mathcal{C}\right|_{X}$, and $A \subseteq Y$ is a puredimensional analytic set. Let $a:=[A] \in D(Y)$ be the Douady point of $A$. Let $D_{\alpha}$ be an irreducible component of $D(Y)$ such that $a \in D_{\alpha}$. Now $Z_{\alpha} \subseteq D_{\alpha} \times Y$ is named by a predicate, and hence $A=\left(Z_{\alpha}\right)_{a}$ is definable over $a$ in $\left.\mathcal{C}\right|_{X}$. On the other hand, if $\sigma \in \operatorname{Aut}\left(\left.\mathcal{C}\right|_{X}, s: s \in S\right)$ and $\sigma(A)=A$, then $\left(Z_{\alpha}\right)_{\sigma(a)}=A$. As $\sigma$ fixes $U(Y, \alpha)$, the sheaf-theoretic fibre of $Z_{\alpha}$ above $\sigma(a)$ is also reduced. So $\sigma(a)$ is the Douady point of $A$ and $\sigma(a)=a$. All pure-dimensional analytic sets have codes.

Suppose $B \subseteq Y$ is an analytic set with irreducible components $A_{1}, \ldots, A_{n}$. Let $a_{i}$ be a code for $A_{i}$, and $b$ a code for the finite (and hence pure-dimensional analytic) set $\left\{a_{1}, \ldots, a_{n}\right\}$. Then $b$ is a code for $B$. Also, if $A$ is irreducible analytic, $B \subset A$ is a proper analytic subset, $a$ is a code for $A$, and $b$ is a code for $B$, then it is not hard to see that $a b$ is a code for $A \backslash B$. Finally, by quantifier elimination (in $\mathcal{A}$ ) every definable set $F$ in $\left.\mathcal{C}\right|_{X}$ can be expressed irredundantly as a finite union of sets of the form $A \backslash B$ as above. Each of these have codes. As before, the code for this finite set of codes is a code for $F$. This completes the proof of the lemma.

Ranging over all Kähler-type varieties $X$, Lemma 4.5 yields Proposition 4.4.

\section{Relative Algebraic Reductions}

The purpose for considering essentially saturated compact complex varieties is that under such hypotheses there is a natural (from the model-theoretic point of view) notion of "generic point"; and hence of "generic member" of a definable family of analytic sets. These notions of genericity often produce some interesting uniform behaviour. For the remainder of this section we discuss one such example. Namely, we will use model-theoretic methods to give a proof of the fact that relative algebraic reductions exist for fibre spaces of Kähler-type varieties (Campana [4], Fujiki [12]). Relative algebraic reductions played an important role in Fujiki's analysis of the structure of Kähler-type varieties in [11].

We begin by recalling some definitions. If $f: X \rightarrow Y$ is a meromorphic surjection of compact complex varieties, then by a fibre of $f$ we mean a fibre of its graph under the co-ordinate projection $\Gamma(f) \rightarrow Y$. Note that after a natural identification, this coincides with the usual notion when $f$ is holomorphic. We say that $f$ is a meromorphic fibre space if over a non-empty Zariski open subset of $Y$ the fibres of $f$ are irreducible. We simply say that $f$ is a fibre space if in addition $f$ is holomorphic.

Suppose $X$ is a compact complex variety. The meromorphic function field of $X$, denoted by $\mathbb{C}(X)$, is a finitely generated extension of $\mathbb{C}$ whose transcendence degree over $\mathbb{C}$ is bounded by the dimension of $X$. This transcendence degree is called the algebraic dimension of $X$, and is denoted by a $(X)$. A compact complex variety is Moishezon if its dimension is equal to its algebraic dimension. 
An algebraic reduction of $X$ is a meromorphic fibre space $f: X \rightarrow V$, such that $V$ is a Moishezon variety and $\operatorname{dim} V=\mathrm{a}(X)$. An algebraic reduction always exists. We sketch a construction from [23]. Let $V$ be a projective manifold whose rational function field is $\mathbb{C}(X)$. Then $\operatorname{dim} V=\mathrm{a}(X)$. Let $\mathbb{C}\left[P_{0}, \ldots P_{n}\right]$ be a homogeneous co-ordinate ring for $V$ such that $f_{1}=\frac{P_{1}}{P_{0}}, \ldots, f_{n}=\frac{P_{n}}{P_{0}}$ generate $\mathbb{C}(X)$. Viewing $f_{1}, \ldots, f_{n}$ as meromorphic functions on $X$, we obtain an induced meromorphic surjection $f: X \rightarrow V$ given by $x \mapsto\left(1: f_{1}(x): \cdots: f_{n}(x)\right)$; such that $f^{*}: \mathbb{C}(V) \rightarrow$ $\mathbb{C}(X)$ is an isomorphism. Now let $h: M \rightarrow \Gamma(f)$ be a resolution of the graph of $f$, so $M$ is a compact complex manifold and $h$ is a modification. Let $g: M \rightarrow V$ be the holomorphic map obtained by composing $h$ with the co-ordinate projection $\Gamma(f) \rightarrow V$. Then $g^{*}: \mathbb{C}(V) \rightarrow \mathbb{C}(M)$ is an isomorphism. By considering a Stein factorisation of $g$ we see that the fibres of $g$ must be connected and hence irreducible (as $M$ is smooth). It follows that the fibres of $\Gamma(f) \rightarrow V$ are irreducible and $f$ is a meromorphic fibre space.

It follows from the above construction that $X$ is Moishezon if and only if it is bimeromorphic to a projective manifold. Moreover, any meromorphic surjection of $X$ onto a Moishezon variety factors meromorphically through an algebraic reduction of $X$. Hence, up to bimeromorphic equivalence, algebraic reductions are unique.

Suppose $f: X \rightarrow Y$ is a fibre space. A relative algebraic reduction of $f$ is a commutative diagram:

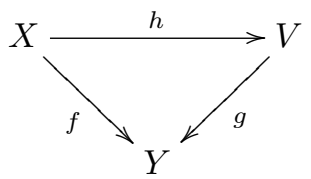

where $V$ is a compact complex variety, $g: V \rightarrow Y$ is a fibre space, $h: X \rightarrow V$ is a meromorphic map over $Y$, and there is a countable intersection of non-empty Zariski open subsets, $Q \subseteq Y$, such that for all $q \in Q, h_{q}: X_{q} \rightarrow V_{q}$ is an algebraic reduction of $X_{q}$. Note that while $Q$ is not itself required to be Zariski open, it is (by Baire category) a dense set of points in $Y$.

As mentioned earlier, Campana [4] and Fujiki [12] have shown that relative algebraic reductions exist when $X$ and $Y$ are Kähler-type varieties. In fact they show something somewhat stronger: a relative algebraic reduction can be found so that $V_{q}$ as above is a projective variety (rather than just bimeromorphic to one). In any case, we give a model-theoretic explanation:

Proposition 4.6. Suppose $f: X \rightarrow Y$ is a fibre space of Kähler-type varieties. Then a relative algebraic reduction of $f$ exists.

Proof. Just as in the proof of Proposition 4.4, we let $\left\{X_{i}: i \in \omega\right\}$ be the smallest collection of sorts from $\mathcal{C}$ containing $X, Y$, and $\mathbb{P}$, that is closed under cartesian products as well as taking irreducible components of restricted Douady spaces. Let $\mathcal{C}^{\circ}$ be the full reduct of $\mathcal{C}$ to these sorts: there is a predicate for each component of the universal family of each cartesian product of the $X_{i}$ 's. Moreover, let $S$ be a countable set of parameters such that if $D_{\alpha}$ is any irreducible component of the restricted Douady space of some product of the $X_{i}$ 's, and $U_{\alpha} \subseteq D_{\alpha}$ is the (Zariski open) set of points above which the universal family has a reduced fibre, then $U_{\alpha}$ is definable in $\mathcal{C}^{\circ}$ over $S$. Adding countably more elements to $S$ we may assume that $S=\operatorname{acl}(S)$ and that $f: X \rightarrow Y$ is defined over $S$. 
We will work in the structure $\left(\mathcal{C}^{\circ}, s: s \in S\right)$, which is saturated and eliminates imaginaries (Lemma 4.5). Note that automorphisms of this structure preserve properties such as being irreducible analytic or being Moishezon (it is for the latter that we have included the sort $\mathbb{P}$ ). In particular, the conjugate of an algebraic reduction of $X$ under an automorphism $\sigma$ is an algebraic reduction of $\sigma(X)$.

Let $c \in Y$ be a generic point of $Y$ over $S$ : a realisation of the type saying $x \in Y$ but $x \notin A$ for any $S$-definable proper analytic set $A \subset Y$. Such a type is realised in $Y$ by saturation. We claim:

Lemma 4.7. There is an $(S \cup\{c\})$-definable algebraic reduction of $X_{c}$ in $\mathcal{C}^{\circ}$.

Proof. Let $X_{c} \rightarrow W$ be any algebraic reduction and $\Gamma \subseteq W \times X_{c}$ its graph. Applying Hironaka's flattening theorem as in the proof of Lemma 3.4, there is a non-empty Zariski open subset $U \subseteq W$ such that $\Gamma_{U} \rightarrow U$ is flat, has reduced fibres, and the Douady map associated to $\Gamma_{U} \rightarrow U$ extends to a meromorphic surjection from $W$ to an irreducible analytic set $W^{\prime} \subseteq D\left(X_{c}\right)$; which we denote by $\phi: W \rightarrow W^{\prime}$.

Moreover, since $\Gamma$ is the graph of a meromorphic map from $X_{c}$ to $W$, we may assume, after shrinking $U$ if necessary, that the fibres of $\Gamma \rightarrow W$ over $U$ are distinct as analytic subsets of $X_{c}$. It follows that the Douady map $\left.\phi\right|_{U}$ is injective, and hence $\phi$ is a bimeromorphism. Letting $\Gamma^{\prime}:=Z\left(X_{c}\right) \cap\left(W^{\prime} \times X_{c}\right)$ we have that $\phi \times$ id is a bimeromorphism from $\Gamma$ to $\Gamma^{\prime}$ that lifts $\phi$. In diagrams,

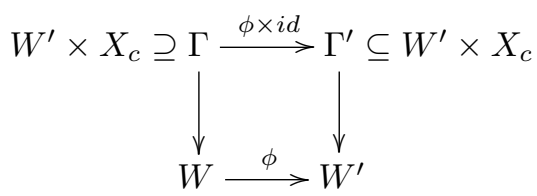

Hence $\Gamma^{\prime}$ is also the graph of an algebraic reduction of $X_{c}, h^{\prime}: X_{c} \rightarrow W^{\prime}$.

There is a natural identification of $D\left(X_{c}\right)$ with an analytic subspace of $D(X)$ (given by a Douady map). Under this identification, $Z\left(X_{c}\right)$ is the restriction of $Z(X)$ to $D\left(X_{c}\right)$. Hence for some irreducible component $D_{\alpha}$ of $D(X), W^{\prime} \subseteq D_{\alpha}$ and $\Gamma^{\prime}=Z_{\alpha} \cap\left(W^{\prime} \times X\right)$. As $D_{\alpha}$ is a sort of $\mathcal{C}^{\circ}, h^{\prime}: X_{c} \rightarrow W^{\prime}$ is a definable algebraic reduction of $X_{c}$ in $\mathcal{C}^{\circ}$. We claim that $h^{\prime}$ is $(S \cup\{c\})$-definable.

Let $\sigma$ be an automorphism of $\mathcal{C}^{\circ}$ that fixes $S \cup\{c\}$ pointwise. Then $\sigma\left(W^{\prime}\right) \subseteq D_{\alpha}$ and $\sigma\left(\Gamma^{\prime}\right)=Z_{\alpha} \cap\left(\sigma\left(W^{\prime}\right) \times X\right)$. On the other hand, $\sigma\left(\Gamma^{\prime}\right) \subseteq \sigma\left(W^{\prime}\right) \times X_{c}$ is the graph of another algebraic reduction $h^{\prime \sigma}: X_{c} \rightarrow \sigma\left(W^{\prime}\right)$. The uniqueness of algebraic reductions implies that there is a bimeromorphism $m: W^{\prime} \rightarrow \sigma\left(W^{\prime}\right)$ such that $m \circ h^{\prime}=h^{\prime \sigma}$. We have non-empty Zariski open sets $A \subseteq W^{\prime}$ and $B \subseteq \sigma\left(W^{\prime}\right)$ such that $m: A \rightarrow B$ is an isomorphism and for all $a \in A, \Gamma_{a}^{\prime}=\sigma\left(\Gamma^{\prime}\right)_{m(a)}$. That is, $\left(Z_{\alpha}\right)_{a}=\left(Z_{\alpha}\right)_{m(a)}$ for all $a \in A$.

By our choice of $S, \sigma$ fixes the (dense Zariski open) set of points in $D_{\alpha}$ above which the fibres of $Z_{\alpha}$ are reduced. Moreover, this set has a non-empty intersection with $W^{\prime}$ (the fibres of $\Gamma_{U} \rightarrow U$ are reduced). Hence it also has a non-empty intersection with $\sigma\left(W^{\prime}\right)$. We may therefore assume, after possibly shrinking $A$ further, that for each $a \in A$, the fibres of $Z_{\alpha}$ over $a$ and $m(a)$ are reduced. So for each $a \in A, a$ is the Douady point of $\Gamma_{a}^{\prime}=\left(Z_{\alpha}\right)_{a}$ and $m(a)$ is the Douady point of $\sigma\left(\Gamma^{\prime}\right)_{m(a)}=\left(Z_{\alpha}\right)_{m(a)}$. The uniqueness of Douady points implies that $m$ is the identity and $A=B$. Taking Zariski closures, $W^{\prime}=\sigma\left(W^{\prime}\right)$ and so $\Gamma^{\prime}=\sigma\left(\Gamma^{\prime}\right)$. That is, every automorphism of $\mathcal{C}^{\circ}$ fixing $S \cup\{c\}$ pointwise fixes the graph of $h^{\prime}$ setwise. By saturation, $h^{\prime}$ is $(S \cup\{c\})$-definable, as desired. 
Let $h^{\prime}: X_{c} \rightarrow W^{\prime}$ be the $(S \cup\{c\})$-definable algebraic reduction of $X_{c}$ in $\mathcal{C}^{\circ}$ obtained above. Let $a \in X_{c}$ be generic in $X_{c}$ over $(S \cup\{c\})$ and $b:=h^{\prime}(a)$. Let $V$ be the $S$-locus of $(c, b)$ : the smallest analytic set defined over $S$ that contains $(c, b)$. Note that an irreducible component of $V$ has finitely many $S$-conjugates and hence (by saturation and elimination of imaginaries) is defined over $\operatorname{acl}(S)$. But $\operatorname{acl}(S)=S$ and so $V$ is irreducible. Let $g: V \rightarrow Y$ be the co-ordinate projection. Note that $V_{c}=W^{\prime}$ since $b$ is generic in $W^{\prime}$ over $(S \cup\{c\})$. Moreover, as $c \in Y$ is generic over $S, h^{\prime}: X_{c} \rightarrow V_{c}$ extends to a meromorphic map $h: X \rightarrow V$ over $Y$, such that $h_{c}=h^{\prime}$. Let $Q \subseteq Y$ be the set of realisations of $\operatorname{tp}(c / S)$. If $q \in Q$ then by saturation $q$ is an $S$-conjugate of $c$, and hence $h_{q}: X_{q} \rightarrow V_{q}$ is an algebraic reduction. Since $\operatorname{tp}(c / S)$ is the generic type of $Y$ (and by quantifier elimination), $Q$ contains a countable intersection of non-empty Zariski open subsets of $Y$. Hence $h$ is a relative algebraic reduction of $f$. This completes the proof of Proposition 4.6.

\section{APPENDIX}

The following sufficient condition for saturation was used in the proof of Proposition 2.3 to avoid the Continuum Hypothesis. The argument presented below was pointed out to the author by Anand Pillay and is related to the notion of "gross" models from [16]. Notions from geometric stability theory are involved, for which we suggest [21] as a general reference.

Proposition 5.1. Suppose $\mathcal{L}$ is a countable language and $T$ is an $\mathcal{L}$-theory such that for all $n \in \omega$ every $n$-type in $T$ is of finite $U$-rank. Suppose $\kappa$ is an uncountable cardinal and $N$ is a model of $T$ that satisfies the following strengthening of $\omega_{1}$-saturation: every non-algebraic complete type in $T^{\mathrm{eq}}$ over countably many parameters from $N^{\mathrm{eq}}$ has $\kappa$-many realisations in $N^{\mathrm{eq}}$. Then $N$ is $\kappa$-saturated.

Proof. We work in a saturated elementary extension $M$ of $N$. We need to show that for every set $B \subset N$ of cardinality less than $\kappa$, every complete type $q(x)$ over $B$ has a realisation in $N$. We proceed by induction on the $U$-rank of $q$. This is clear for the $U$-rank 0 case since every algebraic type is realised in $N$.

Suppose the $U$-rank of $q$ is $n+1$. We pass to $M^{\text {eq }}$. Since $\operatorname{acl}^{\mathrm{eq}}(B) \subset N^{\mathrm{eq}}$, and the cardinality of $\operatorname{acl}^{\mathrm{eq}}(B)$ is also less than $\kappa$ (as the language is countable), we may assume that $B=\operatorname{acl}^{\mathrm{eq}}(B)$.

There is a finite subset of $B$ over which $q$ does not fork. Taking its algebraic closure in $M^{\mathrm{eq}}$, there is a countable set $A \subseteq B$, such that the restriction of $q$ to $A, p(x)$, is stationary and $q$ does not fork over $A$. In particular, $q$ is the unique nonforking extension of $p$ to $B$. Any realisation of $p$ in $N$ that is independent from $B$ over $A$ would thus produce the desired realisation of $q$ in $N$.

As $U(p)<\omega, p$ is not orthogonal to some stationary $U$-rank 1 type in $M^{\text {eq }}$. That is, for some countable set $C \subset M^{\text {eq }}$, containing $A$, there exist: a $U$-rank 1 type $r(y)$ in $M^{\text {eq }}$ over $C$, a realisation $b$ of $r$, and a realisation $a$ of the nonforking extension of $p$ to $C$; such that $a$ and $b$ are dependent over $C$. By the $\omega_{1}$-saturation of $N$, we can find $C, a$, and $b$ in $N^{\text {eq }}$. Increasing $A$ (and $B$ ) by countably many elements if necessary, we may assume that $C=A$. That is, $a$ is a realisation of $p(x)$ in $N, b$ is a realisation of $r(y)$ in $N^{\mathrm{eq}}$, and $a$ forks with $b$ over $A$.

Let $s(x, y)=\operatorname{tp}(a b / A)$, let $s^{\prime}(x, y)$ be the nonforking extension of $s$ to $B$, and let $r^{\prime}(y)$ be the nonforking extension of $r$ to $B$. Suppose $a^{\prime} b^{\prime}$ is a realisation of $s^{\prime}$ in 
$N^{\text {eq }}$. Then $a^{\prime}$ realises $p$ and is independent from $B$ over $A$ (as $a^{\prime} b^{\prime}$ is independent from $B$ over $A$ ). So $a^{\prime}$ is a realisation of $q$ in $N$, and we are done. It remains to show that $s^{\prime}$ is realised in $N^{\text {eq }}$.

We first find a realisation of $r^{\prime}$ in $N^{\text {eq }}$. As $r^{\prime}$ is the unique nonforking extension of $r$ to $B$, we need a realisation of $r$ in $N^{\text {eq }}$ that is independent from $B$ over $A$. But since $U(r)=1$, any realisation of $r$ that is not algebraic over $B$, would be independent from $B$ over $A$. Since $B=\operatorname{acl}^{\mathrm{eq}}(B)$, all we require is a realisation of $r$ in $N^{\mathrm{eq}}$ that is not contained in $B$. But $|B|<\kappa$, and by assumption $r$ has $\kappa$-many realisations in $N^{\text {eq }}$. Hence there must be some $b^{\prime}$ from $N^{\text {eq }}$ that realises $r^{\prime}$.

As $a$ and $b$ were dependent over $A, s(x, b)$ is a forking extension of $p$ to $A b$. Since $b$ and $b^{\prime}$ realise the same complete type over $A$ (namely $r(y)$ ), $s\left(x, b^{\prime}\right)$ is a forking extension of $p$ to $A b^{\prime}$. Finally, $s^{\prime}\left(x, b^{\prime}\right)$ extends $s\left(x, b^{\prime}\right)$ from $A b^{\prime}$ to $B b^{\prime}$. By transitivity $s^{\prime}\left(x, b^{\prime}\right)$ is a forking extension of $p$ to $B b^{\prime}$. It follows that $U\left(s^{\prime}\left(x, b^{\prime}\right)\right)<$ $U(p)=U(q)=n+1$. By induction, $s^{\prime}\left(x, b^{\prime}\right)$ has a realisation in $N$, say $a^{\prime}$. That is, $a^{\prime} b^{\prime}$ is a realisation of $s^{\prime}$ in $N^{\mathrm{eq}}$, as desired.

\section{Elimination of Imaginaries}

In [22], Pillay sketches how Grauert's theorem on meromorphic equivalence relations from [14] can in principle be used to show that $\mathcal{A}$ admits elimination of imaginaries. We provide the details of the argument here.

Suppose $X$ is a compact complex manifold. A meromorphic equivalence relation on $X$ is an analytic set $R \subseteq X \times X$ that contains the diagonal, is symmetric, and such that there is a proper analytic set $P \subset X$ such that,

(i) no irreducible component of $R$ is contained in $X \times P$;

(ii) $R^{\circ}:=R \cap(X \backslash P)^{2}$ is an equivalence relation on $X \backslash P$;

(iii) the dimensions of the $R^{\circ}$-classes in $X \backslash P$ are constant; and,

(iv) the co-ordinate projections, $R^{\circ} \rightarrow X \backslash P$, are open maps.

We call $P$ a degeneracy set for $R$.

Proposition 5.2. Suppose $X$ is a compact complex manifold and $E$ is a definable equivalence relation on $X$. Then there exists a meromorphic equivalence relation $R$ on $X$, and a degeneracy set $P$ for $R$, such that $R$ agrees with $E$ outside of $P$.

Proof. We have an irredundant expression $E=\bigcup_{i=0}^{n} S_{i} \backslash Q_{i}$, where for each $i \leq n$, $Q_{i}$ is a proper analytic subset of the irreducible analytic subset $S_{i} \subseteq X \times X$. Since $E$ contains the diagonal, some $S_{i}$ must contain the diagonal and hence must project onto $X$ in both co-ordinates. After re-indexing $\left\{S_{0}, \ldots, S_{n}\right\}$ we may assume that for some $0 \leq m \leq n, S_{i}$ projects onto $X$ in both co-ordinates if and only if $i \leq m$.

Let $R=\bigcup_{i=0}^{m} S_{i}$ and $S=\bigcup_{i=0}^{n} S_{i}$. It is clear that $R$ is an analytic set in $X \times X$ that contains the diagonal. Since reflection across the diagonal is an analytic isomorphism of $X \times X$, the symmetry of $E$ implies that $S$ is symmetric. Moreover, since reflection preserves the property of projecting onto $X$ in both co-ordinates, it will permute $\left\{S_{0}, \ldots, S_{m}\right\}$. So $R$ is symmetric. We will construct a proper analytic subset of $X, P$, such that conditions $(i)-(i v)$ hold and $R$ agrees with $E$ on $X \backslash P$. First of all, notice that the only components of $S$ that are not contained in $R$ are those that project (in one or both directions) onto a proper Zariski closed subset of $X$. Letting $P^{\prime}$ be the union of these projections, we get that $P^{\prime}$ is a proper analytic set in $X$ and that $R$ and $S$ agree on $\left(X \backslash P^{\prime}\right)^{2}$. 
Let $\pi: X \times X \rightarrow X$ be the second co-ordinate projection map. Let $U \subseteq X \backslash P^{\prime}$ be a non-empty Zariski open subset such that for each $i \leq m$, the restriction of $\pi: S_{i} \rightarrow X$ to $U,\left(S_{i}\right)_{U} \rightarrow U$, is open (see [7]). Shrinking $U$ further, we may assume that either $Q_{i} \cap(X \times U)=\emptyset$, or $\left(Q_{i}\right)_{U} \rightarrow U$ is also open. In particular we have that for all $i \leq m$, the fibres of $\left(S_{i}\right)_{U} \rightarrow U$ and $\left(Q_{i}\right)_{U} \rightarrow U$ are pure-dimensional and of constant dimension.

Let $P:=X \backslash U$ and $R^{\circ}:=R \cap(U \times U)$. As $P$ is a proper analytic set in $X$, no component of $R$ is contained in $X \times P$. Moreover, $\pi$ restricts to an open map $R^{\circ} \rightarrow U$, whose fibres are of constant dimension. While we have only been considering the second co-ordinate projection, by symmetry, this is also true of the other co-ordinate projection. All that remains to be shown then, is that $R$ and $E$ agree on $U^{2}$; that is, $R^{\circ}=E \cap(U \times U)$.

Claim: For all $a \in U, E_{a} \cap U$ is Zariski dense in $R_{a}^{\circ}=R_{a} \cap U$. Since $E$ is Zariski dense in $S$, and $R$ and $S$ agree on $U^{2}$ (as $P^{\prime} \subseteq P$ ), $E \cap U^{2}$ is Zariski dense in $R \cap U^{2}$. But this does not imply directly that the same is true fibrewise (which is the content of the claim). Fix $i \leq m$. Since $Q_{i}$ is a proper analytic subset of the irreducible $S_{i}$, there must be some $a \in U$ such that $\operatorname{dim}\left(Q_{i}\right)_{a}<\operatorname{dim}\left(S_{i}\right)_{a}$. But as the fibres of $\left(S_{i}\right)_{U} \rightarrow U$ and $\left(Q_{i}\right)_{U} \rightarrow U$ are of constant dimension, $\operatorname{dim}\left(Q_{i}\right)_{a}<\operatorname{dim}\left(S_{i}\right)_{a}$ for all $a \in U$. As the fibres of $\left(S_{i}\right)_{U} \rightarrow U$ are pure-dimensional, we have that $\left(Q_{i}\right)_{a}$ cannot contain any irreducible component of $\left(S_{i}\right)_{a}$. Hence $E_{a}=S_{a} \backslash Q_{a}$ is Zariski dense in $S_{a}$, for all $a \in U$. Intersecting with $U$ and recalling that $R$ and $S$ agree on $U^{2}$, yields the claim.

Suppose $a \in U$ and $b \in R_{a} \cap U$. Let $c \in E_{a} \cap U$. As $E$ is an equivalence relation, $E_{c}=E_{a}$. By the Claim, it follows that $R_{c} \cap U=R_{a} \cap U$. So $b \in R_{c} \cap U$ and by symmetry $c \in R_{b} \cap U$. What we have shown is that $E_{a} \cap U \subseteq R_{b} \cap U$. But as $R^{\circ} \rightarrow U$ has fibres of constant dimension, $\operatorname{dim}\left(R_{a} \cap U\right)=\operatorname{dim}\left(R_{b} \cap U\right)$. Using the Claim again, we get that $E_{a} \cap U$ and $E_{b} \cap U$ must have a non-empty intersection. Since $E$ is an equivalence relation, $E_{a}=E_{b}$. In particular $b \in E_{a} \cap U$. We have shown that $R_{a} \cap U \subseteq E_{a} \cap U$ for all $a \in U$. The converse is clear. So $R^{\circ}=R \cap(U \times U)=E \cap(U \times U)$, as desired.

The theorem of Grauert referred to above, or rather a somewhat special case of that theorem, asserts the existence of a "generic" quotient of a compact complex manifold by a meromorphic equivalence relation:

Fact 5.3 (Grauert [14]). Suppose $X$ is a compact complex manifold and $R$ is a meromorphic equivalence relation on $X$ with degeneracy set $P$. Then there exist compact complex varieties $X^{\prime}$ and $Q$, a modification $\sigma: X^{\prime} \rightarrow X$, and a holomorphic $\operatorname{map} q: X^{\prime} \rightarrow Q$, such that

(i) for each $s \in Q, \sigma\left(q^{-1}(s)\right) \cap(X \backslash P)$ is either empty or an $R^{\circ}$-class; and,

(ii) the association $s \longmapsto \sigma\left(q^{-1}(s)\right) \cap(X \backslash P)$ yields a bijection between the set $\left\{s \in Q: \sigma\left(q^{-1}(s)\right) \cap(X \backslash P) \neq \emptyset\right\}$ and the set of $R^{\circ}$-classes.

Corollary 5.4. $\operatorname{Th}(\mathcal{A})$ admits elimination of imaginaries.

Proof. By quantifier elimination, resolution of singularities, and induction on dimension, it suffices to prove: If $X$ is a compact complex manifold and $E$ is a definable equivalence relation on $X$, then there exists a non-empty Zariski open subset $U \subseteq X$, a compact complex variety $Q$, and a definable map $f: U \rightarrow Q$, such that $f(a)=f(b)$ if and only if $a E b$. 
By Proposition 5.2 we have a meromorphic equivalence relation on $X, R$, with degeneracy set $P$, such that $R$ agrees with $E$ on the Zariski open subset $U=X \backslash P$. Let $\sigma: X^{\prime} \rightarrow X$ and $q: X^{\prime} \rightarrow Q$ be as in Fact 5.3. We define a function $f: U \rightarrow Q$ by $f(a)=s$ if there exists $x \in q^{-1}(s)$ such that $\sigma(x)=a$. Then $f$ is a definable function, and for all $a, b \in U, f(a)=f(b)$ if and only if $a E b$.

\section{REFERENCES}

[1] J. Baldwin. Fundamentals of Stability Theory. Springer-Verlag, Berlin, 1988.

[2] C. Bănică. Le lieu réduit et le lieu normal d'un morphisme. In Romanian-Finnish Seminar on Complex Analysis (Proc. Bucharest, 1976), volume 743 of Lecture Notes in Math. Springer, Berlin, 1979.

[3] F. Campana. Algébricité et compacité dans l'espace des cycles d'un espace analytique complexe. Mathematische Annalen, 251(1):7-18, 1980.

[4] F. Campana. Réduction algébrique d'un morphisme faiblement Kählerien propre et applications. Mathematische Annalen, 256(2):157-189, 1981.

[5] F. Campana and T. Peternell. Cycle spaces. In H. Grauert, T. Peternell, and R. Remmert, editors, Several Complex Variables VII, volume 74 of Encyclopedia of Mathematical Sciences, pages 319-349. Springer-Verlag, Berlin, 1994.

[6] A. Douady. Le problème des modules pour les sous espaces analytiques compacts d'un espace analytique donné. Annales de l'Institut Fourier (Grenoble), 16:1-95, 1966.

[7] G. Fischer. Complex Analytic Geometry. Springer-Verlag, Berlin, 1976.

[8] A. Fujiki. Closedness of the Douady spaces of compact Kähler spaces. Publication of the Research Institute for Mathematical Sciences, 14(1):1-52, 1978.

[9] A. Fujiki. Countability of the Douady space of a complex space. Japanese Journal of Mathematics (New Series), 5(2):431-447, 1979.

[10] A. Fujiki. On the Douady space of a compact complex space in the category $\mathcal{C}$. Nagoya Mathematical Journal, 85:189-211, 1982.

[11] A. Fujiki. On the structure of compact complex manifolds in C. In Algebraic Varieties and Analytic Varieties, volume 1 of Advanced Studies in Pure Mathematics, pages 231-302. NorthHolland, Amsterdam, 1983.

[12] A. Fujiki. Relative algebraic reduction and relative Albanese map for a fibre space in $\mathcal{C}$. Publications of the Research Institute for Mathematical Sciences, 19(1):207-236, 1983.

[13] A. Fujiki. On the Douady space of a compact complex space in the category $\mathcal{C}$, II. Publications of the Research Institute for Mathemtaical Sciences, 20:461-489, 1984.

[14] H. Grauert. On meromorphic equivalence relations. In A. Howard and P. Wong, editors, Contributions to Several Complex Variables, Aspects of Mathematics, pages 115-147. Vieweg, Wiesbaden, 1986.

[15] H. Hironaka. Flattening theorem in complex-analytic geometry. American Journal of Mathematics, 97:503-547, 1975.

[16] M.C. Laskowski and A. Pillay. Morley's theorem for gross models. Preprint, 2002.

[17] R. Moosa. Contributions to the model theory of fields and compact complex spaces. PhD thesis, University of Illinois, Urbana-Champaign, 2001.

[18] R. Moosa. The model theory of compact complex spaces. To appear in the Proceedings of the Logic Colloquium'01 (Vienna), 2001.

[19] R. Moosa. A nonstandard Riemann existence theorem. Transactions of the American Mathematical Society, 356(5):1781-1797, 2004.

[20] S. Łojasiewicz. Introduction to Complex Analytic Geometry. Birkhäuser Verlag, Basel, 1991. English edition.

[21] A. Pillay. Geometric Stability Theory, volume 32 of Oxford Logic Guides. Oxford Science Publications, Oxford, 1996.

[22] A. Pillay. Some model theory of compact complex spaces. In Workshop on Hilbert's tenth problem: relations with arithmetic and algebraic geometry, volume 270, Ghent, 2000. Contemporary Mathematics.

[23] K. Ueno. Classification Theory of Algebraic Varieties and Compact Complex Spaces, volume 439 of Lecture Notes in Mathematics. Springer-Verlag, Berlin, 1975. 
[24] B. Zilber. Model theory and algebraic geometry. In Proceedings of the 10th Easter Conference on Model Theory, Berlin, 1993.

University of Waterloo, Department of Pure Mathematics, 200 University Avenue West, Waterloo, Ontario, N2L 3G1, Canada

E-mail address: rmoosa@math.uwaterloo.ca 